\title{
Spin excitations of weakly coupled magnetic atoms
}

\author{
E. C. Goldberg ${ }^{1}$ and F. Flores ${ }^{2}$ \\ ${ }^{1}$ Instituto de Física del Litoral and Departamento de Materiales, Facultad de Ingeniería Química, CONICET-UNL, Santa Fe, Argentina \\ ${ }^{2}$ Departamento Física Teórica de la Materia Condensada, Universidad Autónoma de Madrid, Madrid, Spain
}

(Received 16 December 2014; revised manuscript received 18 March 2015; published 10 April 2015)

\begin{abstract}
A theoretical description of the measured differential conductance through magnetic atoms on a $\mathrm{Cu}_{2} \mathrm{~N} / \mathrm{Cu}(100)$ substrate is presented [Otte et al., Phys. Rev. Lett. 103, 107203 (2009)]. In particular, we analyze the case of a weakly coupled $\mathrm{Co} / \mathrm{Fe}$ dimer. The starting point of our model is an ionic Hamiltonian which describes the inelastic electron tunneling excitations and the Kondo resonances as due to atomic spin fluctuations associated with electron cotunneling processes in the tip-atom-surface system. The interaction terms of this Hamiltonian are written in the basis set of the eigenstates of the atomic part, which in the present case includes the crystalline and Zeeman fields and also, a Heisenberg exchange coupling between the two spins. The appropriate Green's functions that define the differential conductance spectra are calculated by means of the equation-of-motion method. We obtain, in this form, a very satisfactory description of the overall experimental findings related to the behavior of the spin state excitations and Kondo resonance structures in the presence of an applied magnetic field.
\end{abstract}

DOI: 10.1103/PhysRevB.91.165408

\section{INTRODUCTION}

Kondo effects in individual atoms [1-4], molecular magnets [5-7], or quantum dots [8-10] have been extensively studied; apart from their basic interest, these systems are contemplated as possible operational components of a quantum computer [11]. Recently, systems with two units, like double quantum dots [12] or two atoms [13], have attracted increasing attention due to the interplay between the Kondo effect of each unit and the possible antiferromagnetic coupling between the spin of the two components [14].

The cases of one or two magnetic atoms deposited on a metal surface have been recently analyzed by means of inelastic tunneling spectroscopy $[1,2,13]$; in these experiments, electrons injected with a scanning tunneling microscopy (STM) tip on single or double atoms create spin-flip processes that reveal themselves in the tip-metal tunneling conductance measured as a function of the bias voltage. In particular, in Refs. [1] and [2] individual $\mathrm{Fe}$ or $\mathrm{Co}$ atoms have been analyzed and shown to have conductance steps associated with those spin-flip processes; for $\mathrm{Co}$, an additional Kondo resonance is also found at the Fermi energy.

Several authors have analyzed this problem using different techniques [14-20]; we have recently published a paper [21] analyzing both the $\mathrm{Fe}$ and the $\mathrm{Co}$ atoms by introducing an ionic Hamiltonian to describe the $d$ electrons of the magnetic atom and their coupling to the metal states. This approach has allowed us to describe the conductance steps seen experimentally, as well as the Kondo resonance observed for the Co atom.

Otte et al. [13]. used inelastic spin excitation spectroscopy with scanning tunneling microscopy for studying interactions in a dimer formed by a Kondo-screened atomic spin (Co) and a magnetic atom $(\mathrm{Fe})$ that has no Kondo resonance. This two-magnetic-impurity system presents an interesting interplay between Kondo screening, exchange coupling, and magnetocrystalline anisotropy. In particular, the inelastic spectra indicated that there is an antiferromagnetic coupling between the spins of both atoms, and that the Kondo resonance of Co was strongly affected by that coupling and reconstituted by the application of a magnetic field having the suitable magnitude and direction.

In this work we discuss the $\mathrm{Fe} / \mathrm{Co}$ dimer, extending our previous approach to this case; basically, our interest is addressed to understanding theoretically how the coupling between the Fe and Co spins modifies the Kondo resonance found for the individual Co atom, as well as the small structures seen in the differential conductance around the Fermi energy. In our model the atomic part of the multiorbital Anderson Hamiltonian, including Zeeman and magnetocrystalline anisotropy terms and also a Heisenberg coupling between atoms, is diagonalized in the basis set of spin configurations of the noninteracting dimer. By assuming the possibility of spin fluctuations in each atom, the interaction part of the Hamiltonian projected over these eigenstates describes the charge exchange between the atoms and the leads as the result of a cotunneling process: in a first step, an electron tunnels from the atom to one of the leads and, in a second step, another electron jumps from the second lead to the atom. This is the first time a theory is presented fully discussing the inelastic tunneling spectroscopy for two coupled magnetic atoms, including both low-energy processes associated with the Kondo resonance and with the spin excitations.

The paper is organized as follows: Sec. II is devoted to present our model and discuss how to obtain its solution, in Sec. III we present our results and discussion, and in Sec. IV, our conclusions.

\section{THEORY AND MODEL}

The interaction between the tip and two atoms deposited on the surface (see Fig. 1) is well described by the multiorbital Anderson Hamiltonian written below:

$$
\begin{aligned}
H= & \sum_{k, \alpha, \sigma} \varepsilon_{k \alpha} \hat{c}_{k \alpha \sigma}^{+} \hat{c}_{k \alpha \sigma}+H_{\text {atom }}^{A}+H_{\text {atom }}^{B}+H_{A-B} \\
& +\sum_{k, \alpha, l, \sigma}\left(V_{k \alpha l}^{A} c_{k \alpha \sigma}^{+} c_{l \sigma}+\text { c.c. }\right) \\
& +\sum_{k, \alpha, L, \sigma}\left(V_{k \alpha L}^{B} c_{k \alpha \sigma}^{+} c_{L \sigma}+\text { c.c. }\right) .
\end{aligned}
$$




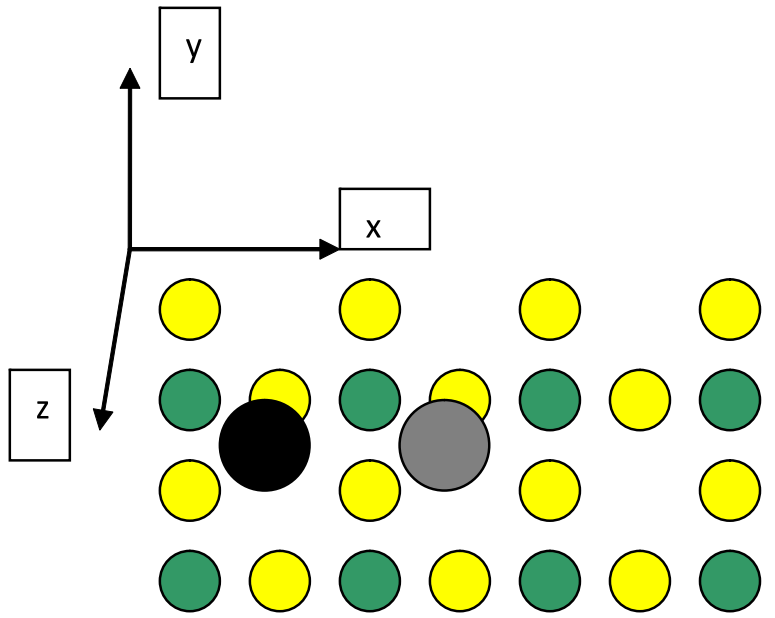

FIG. 1. (Color online) Schematic view of an Fe (black)/Co(gray) dimer on an island of $\mathrm{Cu}_{2} \mathrm{~N} / \mathrm{Cu}(100)$. (Green circles are $\mathrm{Cu}$ atoms and yellow circles are $\mathrm{N}$ atoms.)

The first term in Eq. (1) describes the electrons of the leads, $\hat{c}_{k \alpha \sigma}^{+}\left(\hat{c}_{k \alpha \sigma}\right)$ being the creation (annihilation) operator associated with the state $k \alpha \sigma \quad(\alpha=1,2$ refers to the tip and the metal surface, respectively); $H_{\text {atom }}^{A / B}$ describes the valence electrons of the atoms; $H_{A-B}$ the interaction between atoms; and the last two terms the interaction between the leads and the atoms $A(\mathrm{Co})$ and $B(\mathrm{Fe})$. In these last terms, $V_{k \alpha l}^{A}$ and $V_{k \alpha L}^{B}$ define the coupling between the conduction states $k \alpha \sigma$ and the localized states $l \sigma$ in atom $A$ and $L \sigma$ in atom $B$, respectively, and $\hat{c}_{l \sigma}^{+}\left(\hat{c}_{l \sigma}\right)$ denotes the creation (annihilation) operator of the localized valence electrons in the orbital $l$ with spin $\sigma$. In the particular case of $d$ electrons, $l(L)$ runs over five possibilities which, in the case of no crystalline field effects, correspond to the degenerate orbitals $d_{z}^{2}, d_{x-y}^{2}, d_{x z}, d_{y z}, d_{x y}$. The interaction between the tip and the leads is not included in Eq. (1) because the experimental evidence shows that for $\mathrm{Co}$ on $\mathrm{CuN}$ the Fano line shape is nearly Lorentzian, indicating that the tip-metal hybridization is negligible [2].

The important difference between our model and other works existing in the literature [14-20], resides in the proposal for the atomic part of Hamiltonian (1). The Hamiltonian describing atom $A$ is written as (the case of atom $B$ is completely similar)

$$
H_{\text {atom }}^{A}=H_{\text {atom }}^{A(0)}+H_{p}^{A} .
$$

The first term,

$$
\begin{aligned}
H_{\mathrm{atom}}^{A(0)}= & \sum_{l, \sigma} \varepsilon_{l} \hat{n}_{l \sigma}+\sum_{l} U_{d} n_{l \uparrow} n_{l \downarrow}+\frac{1}{2} \sum_{l \neq l^{\prime}, \sigma} J_{d} n_{l \sigma} n_{l^{\prime}-\sigma} \\
& +\frac{1}{2} \sum_{l \neq l^{\prime}, \sigma}\left(J_{d}-J_{d}^{x}\right) n_{l \sigma} n_{l^{\prime} \sigma} \\
& -\frac{1}{2} \sum_{l \neq l^{\prime}, \sigma} J_{d}^{x} c_{l \sigma}^{+} c_{l-\sigma} c_{l^{\prime}-\sigma}^{+} c_{l^{\prime} \sigma},
\end{aligned}
$$

corresponds to the free atom and it includes the intra-atomic Coulomb interactions $U_{d}$ and $J_{d}$, as well as the intra-atomic exchange interaction $J_{d}^{x}$, all of them assumed to be constants independent of the $l$-orbital index. In Eq. (2) $\hat{n}_{l \sigma}=\hat{c}_{l \sigma}^{+} \hat{c}_{l \sigma}$ and the last term restores the invariance under rotation in spin space.

The second term, $H_{p}^{A}\left(H_{p}^{B}\right)$, includes the magnetocrystalline field and the Zeeman energy term resulting from the external magnetic field $\mathbf{B}$ :

$$
\begin{aligned}
& \hat{H}_{p}^{A}=\mu_{B} \hat{B} \cdot g_{A} \hat{S}+D_{A} \hat{s}_{x}^{2}, \\
& \hat{H}_{p}^{B}=\mu_{B} \hat{B} \cdot g_{B} \hat{S}+D_{B} \hat{S}_{z}^{2}+E_{B}\left(\hat{S}_{x}^{2}-\hat{S}_{y}^{2}\right) .
\end{aligned}
$$

The magnetocrystalline anisotropies experienced by the spins are quantified by the uniaxial $D$ and transversal $E$ anisotropy parameters. In Eq. (3), $\mu_{B}$ is the Bohr magneton and $g_{A}\left(g_{B}\right)$ the $g$ factors of the $A$ and $B$ spins. We are assuming that there is no transverse anisotropy in the case of spin $A\left(E_{A}=0\right)$, and that both spins are referred to a common reference system (see Fig. 1).

The interaction between atoms is assumed to be given by the following Heisenberg coupling [13]:

$$
\hat{H}_{A-B}=J \hat{S} \cdot \hat{s} .
$$

In Eq. (4), positive values of the exchange parameter $J$ indicates antiferromagnetic coupling.

A crucial approximation to analyze the atoms and their interaction with the leads is to assume the exchange interaction $J_{d}^{x}$ to be large enough to make the first Hund-rule operative [22]. Then the atomic lower-energy configurations correspond to the states of maximum electron spin, say $S$. The effect of the atom-leads interactions is to make electrons jump between the leads and the atom, changing the atom spin state between $S$ and $S-1 / 2$. Then we assume for atom $A$ that the most probable electronic configurations are, in the Dirac notation, $|s, m\rangle$ and $\left|s-1 / 2, m^{\prime}\right\rangle$, and for atom $B,|S, M\rangle$ and $\left|S-1 / 2, M^{\prime}\right\rangle$. For instance, in the case of an isolated Fe atom, the ground state $S=2$ is described by means of different kets associated with the degenerate $d$ orbitals $\left|d_{x 2-y 2}, d_{z 2}, d_{z y}, d_{x y}, d_{z x}\right\rangle$ in the following way (also including spin variables):

$$
\begin{aligned}
|S=2, M=2\rangle= & |\uparrow \downarrow, \uparrow 0, \uparrow 0, \uparrow 0, \uparrow 0\rangle, \\
|S=2, M=1\rangle= & \frac{1}{2}\left[\begin{array}{l}
|\uparrow \downarrow, 0 \downarrow, \uparrow 0, \uparrow 0, \uparrow 0\rangle+|\uparrow \downarrow, \uparrow 0,0 \downarrow, \uparrow 0, \uparrow 0\rangle \\
+|\uparrow \downarrow, \uparrow 0, \uparrow 0,0 \downarrow, \uparrow 0\rangle+|\uparrow \downarrow, \uparrow 0, \uparrow 0, \uparrow 0,0 \downarrow,\rangle
\end{array}\right], \\
& \cdots \cdots \cdots \cdots \\
|S=2, M=-2\rangle= & |\uparrow \downarrow, 0 \downarrow, 0 \downarrow, 0 \downarrow, 0 \downarrow\rangle .
\end{aligned}
$$


We project the Hamiltonian (1) on the selected space of configurations defined by the direct product of the electronic configurations of each atom, $|A ; B\rangle=|A\rangle \otimes|B\rangle$. The projection technique allows us to write the atomic part describing the two noninteracting atoms as follows:

$$
\begin{aligned}
H_{\mathrm{atom}}^{A(0)}+H_{\mathrm{atom}}^{B(0)}= & \sum_{\substack{S, M, s, m \\
S^{\prime}, M^{\prime}, s^{\prime}, m^{\prime}}}|S, M ; s, m\rangle\left\langle S, M ; s, m\left|H_{\mathrm{atom}}^{A(0)}+H_{\mathrm{atom}}^{B(0)}\right| S^{\prime}, M^{\prime} ; s^{\prime}, m^{\prime}\right\rangle\left\langle S^{\prime}, M^{\prime} ; s^{\prime}, m^{\prime}\right| \\
= & \sum_{M, m}|S, M ; s, m\rangle\left[\varepsilon_{s}^{(A)}+\varepsilon_{S}^{(B)}\right]\left\langle S, M ; s, m\left|+\sum_{M, m}\right| S, M ; s-1 / 2, m\right\rangle\left[\varepsilon_{s-1 / 2}^{(A)}+\varepsilon_{S}^{(B)}\right]\langle S, M ; s-1 / 2, m| \\
& +\sum_{M, m}|S-1 / 2, M ; s, m\rangle\left[\varepsilon_{s}^{(A)}+\varepsilon_{S-1 / 2}^{(B)}\right]\langle S-1 / 2, M ; s, m| \\
& +\sum_{M, m}|S-1 / 2, M ; s-1 / 2, m\rangle\left[\varepsilon_{s-1 / 2}^{(A)}+\varepsilon_{S-1 / 2}^{(B)}\right]\langle S-1 / 2, M ; s-1 / 2, m|
\end{aligned}
$$

In Eq. (5), $\varepsilon_{s}^{A}$ and $\varepsilon_{S}^{B}$ are the total energies of the atomic configurations $|s, m\rangle$ and $|S, M\rangle$, respectively. The projection of the interaction part of Hamiltonian (1) leads to

$$
\begin{aligned}
H_{\mathrm{int}}= & \sum_{\substack{k, \alpha, \sigma \\
S, M, m}} \hat{c}_{k \alpha \sigma}^{+}|S, M ; s-1 / 2, m-\sigma\rangle\langle S, M ; s-1 / 2, m-\sigma| \sum_{l}\left(V_{k \alpha l}^{A} \hat{c}_{l \sigma}+\text { H.c. }\right)|S, M ; s, m\rangle\langle S, M ; s, m| \\
& +\sum_{\substack{k, \alpha, \sigma \\
M, s, m}} \hat{c}_{k \alpha \sigma}^{+}|S-1 / 2, M-\sigma ; s, m\rangle\langle S-1 / 2, M-\sigma ; s, m| \sum_{L}\left(V_{k \alpha L}^{B} \hat{c}_{L \sigma}+\text { H.c. }\right)|S, M ; s, m\rangle\langle S, M ; s, m|,
\end{aligned}
$$

which finally can be written as

$$
\begin{aligned}
H^{\text {int }}= & (-1)^{2 S} \sum_{k, M, \sigma, m}\left[V_{k \alpha, m, \sigma}^{A} c_{k \sigma}^{+}|S, M ; s-1 / 2, m-\sigma\rangle\langle S, M ; s, m|+\text { H.c. }\right] \\
& +\sum_{k, M, \sigma, m}\left[V_{k \alpha, M, \sigma}^{B} c_{k \sigma}^{+}|S-1 / 2, M-\sigma ; s, m\rangle\langle S, M ; s, m|+\text { H.c. }\right] \\
& +(-1)^{2 S-1} \sum_{k, M, \sigma, m}\left[V_{k \alpha, m, \sigma}^{A} c_{k \sigma}^{+}|S-1 / 2, M ; s-1 / 2, m-\sigma\rangle\langle S-1 / 2, M ; s, m|+\text { H.c. }\right] \\
& +\sum_{k, M, \sigma, m}\left[V_{k \alpha, M, \sigma}^{B} c_{k \sigma}^{+}|S-1 / 2, M-\sigma ; s-1 / 2, m\rangle\langle S, M ; s-1 / 2, m|+\text { H.c. }\right] .
\end{aligned}
$$

The first term of Eq. (6) describes the spin fluctuation of atom $A$ while atom $B$ is in its ground state $|S, M\rangle$; the second one is similar but it represents the spin fluctuation taking place in atom $B$ while atom $A$ is in its ground state $|s, m\rangle$. The third and fourth terms correspond to spin fluctuations in atoms $A$ and $B$, respectively, but with the other atom, $B$ or $A$, having fluctuated to the spin configuration $\left|S-1 / 2, M^{\prime}\right\rangle$ or $\left|s-1 / 2, m^{\prime}\right\rangle$. In general, these last two terms are associated with simultaneous virtual transitions in both atoms and can be neglected, as done from now on. The antisymmetric nature of many fermion wave functions has been taken into account in Eq. (6) through the factors $(-1)^{2 S}$ and $(-1)^{2 S-1}$.

The interaction terms, $V_{k \alpha, m, \sigma}^{A}$ or $V_{k \alpha, M, \sigma}^{B}$, were calculated in a previous work [23] for describing the low-energy excitations of different impurity elements across the $3 d$ row of the periodic table, adsorbed on a metal surface. The following expression was found for the coupling matrix elements $V_{k \alpha, M, \sigma}$ :

$$
V_{k \alpha M \sigma}=\sqrt{\frac{5\left(\mathbf{S}+\mathbf{M}^{*} \operatorname{sgn}(\sigma)\right)}{2 S}} V_{k \alpha},
$$

where $V_{k \alpha}$ defines the strength of the $V_{k \alpha M}$ interaction.

In the following step we diagonalize the atomic Hamiltonian $\left(H_{\mathrm{atom}}^{A}+H_{\mathrm{atom}}^{B}+H_{A-B}\right)$ in the basis set provided by the spin configurations $|S, M ; s, m\rangle,|S, M ; s-1 / 2, m\rangle$, and $|S-1 / 2, M ; s, m\rangle$. The eigenstates for each of the spin configurations are written as

$$
\begin{aligned}
\psi_{i}^{S, s}= & \sum_{M, m} a_{M, m}^{i(S, s)}|S, M ; s, m\rangle \Rightarrow(2 S+1)(2 s+1) \text { states } \\
\psi_{j}^{S, s-1 / 2}= & \sum_{M, m} a_{M, m}^{j(S, s-1 / 2)}|S, M ; s-1 / 2, m\rangle \\
& \Rightarrow(2 S+1)(2 s) \text { states } \\
\psi_{l}^{S-1 / 2, s}= & \sum_{M, m} a_{M, m}^{l(S-1 / 2, s)}|S-1 / 2, M ; s, m\rangle \\
& \Rightarrow(2 S)(2 s+1) \text { states. }
\end{aligned}
$$

The interaction part of the Hamiltonian, Eq. (6), is now projected on the basis set of the eigenstates of the atomic part [Eq. (8)] and finally, the Hamiltonian takes the following form:

$$
\begin{aligned}
H= & \sum_{k \alpha, \sigma} \varepsilon_{k \alpha} n_{k \alpha \sigma}+\sum_{i} E_{i}^{S, s}\left|\psi_{i}^{S, s}\right\rangle\left\langle\psi_{i}^{S, s}\right| \\
& +\sum_{j} E_{j}^{S-1 / 2, s}\left|\psi_{j}^{S-1 / 2, s}\right\rangle\left\langle\psi_{j}^{S-1 / 2, s}\right|
\end{aligned}
$$




$$
\begin{aligned}
& +\sum_{l} E_{l}^{S, s-1 / 2}\left|\psi_{l}^{S, s-1 / 2}\right\rangle\left\langle\psi_{l}^{S, s-1 / 2}\right| \\
& +\sum_{k \alpha, \sigma, i, j}\left[T_{k \alpha, \sigma, j, i}^{S, S-1 / 2,(s)} c_{k \sigma}^{+}\left|\psi_{j}^{S-1 / 2, s}\right\rangle\left\langle\psi_{i}^{S, s}\right|\right. \\
& \left.+T_{k \alpha, \sigma, j, i}^{S, S-1 / 2 *}\left|\psi_{i}^{S, s}\right\rangle\left\langle\psi_{j}^{S-1 / 2, s}\right| c_{k \sigma}\right] \\
& +(-1)^{2 S} \sum_{k \alpha, \sigma, i, l}\left[T_{k \alpha, \sigma, l, i}^{S, s-1 / 2,(S)} c_{k \sigma}^{+}\left|\psi_{l}^{S, s-1 / 2}\right\rangle\left\langle\psi_{i}^{S, s}\right|\right. \\
& \left.+T_{k \alpha, \sigma, l, i}^{s, s-1 / 2 *}\left|\psi_{i}^{S, s}\right\rangle\left\langle\psi_{l}^{S, s-1 / 2}\right| c_{k \sigma}\right],
\end{aligned}
$$

where $E_{i}^{S, s}, E_{j}^{S, s-1 / 2}$, and $E_{l}^{S-1 / 2, s}$ are the eigenvalues corresponding to the following Hamiltonian:

$$
\left(H_{\text {atom }}^{A}+H_{\text {atom }}^{B}+H_{A-B}\right) \psi_{i}^{S, s}=E_{i}^{S, s} \psi_{i}^{S, s} .
$$

The coupling terms introduced in Eq. (9) are given by

$$
\begin{aligned}
T_{k \alpha, \sigma, j, i}^{S, S-1 / 2,(s)} & =\sum_{M, m} V_{k \alpha M \sigma}^{B} a_{M-\sigma, m}^{j(S-1 / 2, s)} a_{M, m}^{i(S, s)}, \\
T_{k \alpha, \sigma, l, i}^{S, s-1 / 2,(S)} & =\sum_{M, m} V_{k \alpha m \sigma}^{A} a_{M, m-\sigma}^{l(S, s-1 / 2)} a_{M, m}^{i(S, s)} .
\end{aligned}
$$

As it was previously mentioned, we identify atom $A$ with Co and atom $B$ with $\mathrm{Fe}$. The case of noninteracting atoms $(J=0)$ has been treated and discussed in a previous work [21]. In that paper we found a good description of the measured conductance spectra as a function of an applied magnetic field for $\mathrm{Fe}$ or Co atoms adsorbed independently on a CuN surface [1,2]. The total spin values $S=2$ and $s=3 / 2$ for describing the lower-energy states of $\mathrm{Fe}$ and $\mathrm{Co}$ atoms, respectively, and their interaction with the metal, were extracted from the experiments. The appropriate spin fluctuations $S=2$ to $S=$ $3 / 2$ for Fe and $s=3 / 2$ to $s=1$ for Co, within a hole picture, were deduced by comparing theory with experiments [21]. In this work, we include the antiferromagnetic coupling $J$ between $\mathrm{Fe}$ and $\mathrm{Co}$ atoms proposed by Otte et al. [13], for explaining the features observed in the conductance spectra measured with the tip positioned on either the Fe or the Co atom of a dimer structure.

\section{A. Green's function technique and equation-of-motion method}

The Keldysh Green's functions [24] are appropriate for treating out-of-equilibrium problems as the one posed by the inelastic tunneling current effects through the magnetic atom. For the sake of simplicity, we will only discuss the case of $\mathrm{Fe}$ (extension to the Co case is immediate by changing $S$ by $s$ and vice versa). A convenient solution of Hamiltonian (9) can be obtained by using the following Green's functions:

$$
\begin{aligned}
G_{i j, l q}^{S, S-1 / 2,(s)}\left(t, t^{\prime}\right)= & i \Theta\left(t^{\prime}-t\right)\left\langle\left\{| \psi _ { i } ^ { S , s } \rangle \left\langle\left.\psi_{j}^{S-1 / 2, s}\right|_{t^{\prime}} ;\right.\right.\right. \\
& \left.\left|\psi_{q}^{S-1 / 2, s}\right\rangle\left\langle\left.\psi_{l}^{S, s}\right|_{t}\right\}\right\rangle
\end{aligned}
$$

and

$$
F_{i j, l q}^{S, S-1 / 2,(s)}\left(t, t^{\prime}\right)=i\left\langle\left[\left|\psi_{i}^{S, s}\right\rangle\left\langle\left.\psi_{j}^{S-1 / 2, s}\right|_{t^{\prime}} ; \mid \psi_{q}^{S-1 / 2, s}\right\rangle\left\langle\left.\psi_{l}^{S, s}\right|_{t}\right]\right\rangle,\right.
$$

where \{\}$/[]$ indicates the anticonmutator/conmutator of the corresponding operators defined in $t^{\prime}$ and $t$, and the function $F$ is the specific Green's function yielding the nonequilibrium properties of the system. We should mention that, in the hole picture we are using in this work, the operator $\left|\psi_{q}^{S-1 / 2, s}\right\rangle\left\langle\left.\psi_{l}^{S, s}\right|_{t}\right.$ (or $\left|\psi_{i}^{S, s}\right\rangle\left\langle\left.\psi_{j}^{S-1 / 2, s}\right|_{t^{\prime}}\right.$ ) is associated with the creation at time $t$ (annihilation at time $t^{\prime}$ ) of one electron in the atomic state $\left|\psi_{i}^{S, s}\right\rangle\left(\left\langle\psi_{j}^{S-1 / 2, s}\right|\right)$, the atom jumping to $\left|\psi_{q}^{S-1 / 2, s}\right\rangle\left(\left|\psi_{i}^{S, s}\right\rangle\right)$. This is reminiscent of the creation operators used to define the conventional Green's functions; Eqs. (11) and (12) can be considered as a generalization of the usual Green's functions to the Hamiltonian we analyze in this paper. Notice, however, that the new operators $\left|\psi_{q}^{S-1 / 2, s}\right\rangle\left\langle\left.\psi_{l}^{S, s}\right|_{t}\right.$ and $\left.\mid \psi_{i}^{S, s}\right\rangle\left\langle\left.\psi_{j}^{S-1 / 2, s}\right|_{t^{\prime}}\right.$ do not satisfy the conmutation rules of the conventional fermionic operators. The properties related to the orthonormality of the electronic configurations have to be taken into account when one is working with these projection operators. For a full discussion of the properties associated with the operators $\left|\chi_{p}\right\rangle\left\langle\chi_{q}\right|$, see Ref. [25].

The Green's functions (11) and (12) are calculated using the equation of motion method up to second order in the interaction $T_{k \alpha, \sigma, j, i}^{S, S-1 / 2,(s)}\left(T_{k \alpha, \sigma, j, i}^{s, s-1 / 2,(S)}\right)$. In this approach (see Ref. [21]) the equation of motion for, say $G_{i j, l q}^{S, S-1 / 2,(s)}\left(t, t^{\prime}\right)$, yields new Green's functions of higher order; these Green's functions are calculated again using the corresponding equation of motion, generating other Green's functions that are closed up in a consistent way up to second order in $T_{k \alpha, \sigma, j, i}^{S, S-1 / 2,(s)}\left(T_{k \alpha, \sigma, j, i}^{S, s-1 / 2,(S)}\right)$. We stress that the equation of motions associated with the higher-order Green's functions incorporate, in the consistent solution for the initial, the two-particle interaction effects that describe the inelastic electron tunneling excitations of the coupled magnetic atoms. This approach yields the following advanced diagonal Green's function $G_{i j, i j}^{S, S-1 / 2,(s)}(\omega)$ :

$$
\begin{aligned}
& {\left[\tilde{\omega}-E_{i}^{S, s}+E_{j}^{S-1 / 2, s}-\sum_{k \alpha, \sigma} \frac{\left|T_{k \alpha, \sigma, j, i}^{S, S-1 / 2,(s) *}\right|^{2}}{\tilde{\omega}-\varepsilon_{k \alpha}}\right.} \\
& {\left[-\sum_{k \alpha, \sigma, p \neq j} \frac{\left|T_{k \alpha, \sigma, p, i}^{S, S-1 / 2,(s) *}\right|^{2}\left\langle 1-n_{k \alpha \sigma}\right\rangle}{\tilde{\omega}-\varepsilon_{k \alpha}-E_{p}^{S-1 / 2, s}+E_{j}^{S-1 / 2, s}}-\sum_{k \alpha, \sigma, p \neq i} \frac{\left|T_{k \alpha, \sigma, j, p}^{S, S-1 / 2,(s) *}\right|^{2}\left\langle n_{k \alpha \sigma}\right\rangle}{\tilde{\omega}-\varepsilon_{k \alpha}-E_{i}^{S, s}+E_{p}^{S, s}}-\sum_{k, \sigma, p} \frac{\left|T_{k \alpha, \sigma, p, i}^{S, s-1 / 2,(S) *}\right|^{2}\left\langle 1-n_{k \sigma}\right\rangle}{\tilde{\omega}-\varepsilon_{k}-E_{p}^{S, s-1 / 2}+E_{j}^{S-1 / 2, s}}\right]^{G_{i j, i j}^{S, S-1 / 2,(s)}}} \\
& =\left\langle n_{i}^{S, s}+n_{j}^{S-1 / 2, s}\right\rangle-\sum_{k \alpha, \sigma, p \neq i} \frac{T_{k \alpha, \sigma, j, p}^{S, S-1 / 2,(s)}\left\langle\mid \psi_{p}^{S, s}\right\rangle\left\langle\psi_{j}^{S-1 / 2, s} \mid c_{k \alpha \sigma}\right\rangle}{\tilde{\omega}-\varepsilon_{k \alpha}-E_{i}^{S, s}+E_{p}^{S, s}} \\
& +\sum_{k \alpha, \sigma, p \neq j} \frac{T_{k \alpha, \sigma, p, i}^{S, S-1 / 2,(s)}\left\langle\mid \psi_{i}^{S, s}\right\rangle\left\langle\psi_{p}^{S-1 / 2, s} \mid c_{k \alpha \sigma}\right\rangle}{\tilde{\omega}-\varepsilon_{k \alpha}-E_{p}^{S-1 / 2, s}+E_{j}^{S-1 / 2, s}}+\sum_{k, \sigma, p} \frac{T_{k, \sigma, p, i}^{S, s-1 / 2,(S)}\left\langle\mid \psi_{i}^{S, s}\right\rangle\left\langle\psi_{p}^{S, s-1 / 2} \mid c_{k \sigma}\right\rangle}{\tilde{\omega}-\varepsilon_{k}-E_{p}^{S, s-1 / 2}+E_{j}^{S-1 / 2, s}} .
\end{aligned}
$$


In Eq. (13), $\varpi=\omega-i \eta$ with $\eta \rightarrow 0$ and $\left\langle n_{k \alpha \sigma}\right\rangle=f_{\alpha<}(\omega)$ is the Fermi distribution function for the $k \alpha$ states $(\alpha=1$ is the tip and $\alpha=2$ the lead). The probabilities of occurrence of the different spin configurations are $n_{i}^{S, s}=\left\langle\mid \psi_{i}^{S, s}\right\rangle\left\langle\psi_{i}^{S, s} \mid\right\rangle$ and $n_{j}^{S-1 / 2, s}=\left\langle\mid \psi_{j}^{S-1 / 2, s}\right\rangle\left\langle\psi_{j}^{S-1 / 2, s} \mid\right\rangle$.

The crossed terms such as $\left\langle\mid \psi_{i}^{S, s}\right\rangle\left\langle\psi_{p}^{S-1 / 2, s} \mid c_{k \sigma}\right\rangle$ are calculated by their expressions, valid in the equilibrium and within a second order in the interaction $T_{k \alpha, \sigma, p, i}^{S, S-1 / 2(s)}$ :

$$
\begin{aligned}
& \left\langle\mid \psi_{i}^{S, s}\right\rangle\left\langle\psi_{p}^{S-1 / 2, s} \mid c_{k \sigma}\right\rangle \\
& =\frac{1}{\pi} T_{k \alpha, \sigma, p, i}^{* S, S-1 / 2(s)} \int_{-\infty}^{\infty} d \omega^{\prime} f_{\prec}\left(\omega^{\prime}\right) \operatorname{Im} \frac{G_{i p, i p}^{S, S-1 / 2(s)}\left(\omega^{\prime}\right)}{\tilde{\omega}^{\prime}-\varepsilon_{k}} .
\end{aligned}
$$

In Eq. (13), the self-energy terms $\sum_{k \alpha, \sigma, p} \frac{\left|T_{k \alpha, \sigma, j, p}^{S, S-1 / 2,(s) *}\right|^{2}\left\langle n_{k \alpha \sigma}\right\rangle}{\tilde{\omega}-\varepsilon_{k \alpha}-E_{i}^{S, s}+E_{p}^{S, s}}$ account for the Kondo resonance in the case of a degenerated ground state and also for the conductance steps associated with transitions to excited configurations $\left|\psi_{p}^{S, s}\right\rangle$ (only the ground-state occupation is significant at this very low temperature equal to $0.5 \mathrm{~K}$ ). The self-energy terms $\sum_{k \alpha, \sigma, p \neq j} \frac{\left|T_{k \alpha, \sigma, p, i}^{S, S-1 / 2,(s) *}\right|^{2}\left\langle 1-n_{k \alpha \sigma}\right\rangle}{\tilde{\omega}-\varepsilon_{k \alpha}-E_{p}^{S-1 / 2, s}+E_{j}^{S-1 / 2, s}}$ are associated with excitations between the spin configurations to which the atom has fluctuated, and these appear in the conductance spectrum as depletions, because of the factor $\left\langle 1-n_{k \alpha \sigma}\right\rangle$, when the transition occurs between degenerated states $\left|\psi_{p}^{S-1 / 2, s}\right\rangle$ and $\left|\psi_{j}^{S-1 / 2, s}\right\rangle$.

The self-energy terms whose denominators have the energy difference $\left(E_{p}^{S, s-1 / 2}-E_{j}^{S-1 / 2, s}\right)$ represent an interaction with the Co atom mediated by the substrate band. They are practically negligible in the case of the Fe-Co dimer; however, their contribution may be important for homogeneous dimers such as Co-Co or Fe-Fe.

The Green's function (13) will be used below to calculate the tunneling current when the tip is positioned over the $\mathrm{Fe}$ atom. The tip-Fe current $I_{\mathrm{Fe}}^{\sigma}$, given by

$$
\left.I_{\mathrm{Fe}}^{\sigma}=-\frac{2 e}{\hbar} \operatorname{Im} \sum_{k, i, j} T_{k 1 j i}^{*}|| \psi_{i}^{S, s}\right\rangle\left\langle\psi_{j}^{S-1 / 2, s} \mid c_{k 1 \sigma}\right\rangle,
$$

takes into account the transfer processes between the $k \sigma$ electrons of the tip and the magnetic atom. This equation can be written in terms of $G$ and $F$ as follows [21]:

$$
\begin{aligned}
\frac{I_{\mathrm{Fe}}^{\sigma}}{2 e / h}= & \frac{1}{2} \sum_{i, j} \int_{-\infty}^{\infty} d \varepsilon \Gamma_{1 \sigma j i}^{S, S-1 / 2(s)}(\varepsilon) \operatorname{Im} \\
& \times\left[F_{i j, i j}^{S, S-1 / 2(s)}(\varepsilon)-2\left(2 f_{1<}(\varepsilon)-1\right) G_{i j, i j}^{S, S-1 / 2(s)}(\varepsilon)\right],
\end{aligned}
$$

$\underset{\text { where }}{S, S-1 / 2(s)} \Gamma_{1 \sigma j i}^{S, S-1 / 2(s)}(\varepsilon)=\pi \sum_{k}\left|T_{k 1 \sigma j i}^{S, S-1 / 2(s)}\right|^{2} \delta\left(\varepsilon-\varepsilon_{k 1}\right)=$ $\eta_{\sigma j i}^{S, S-1 / 2(s)} \Gamma_{1(\mathrm{Fe})}$.

In Eq. (16), $I_{\mathrm{Fe}}^{\sigma}=\sum_{i, j} I_{1 i j}^{\sigma}, I_{1 i j}^{\sigma}$ representing a partial current associated with the $(i, j)$ channel. Current conservation for each channel, $I_{1 i j}^{\sigma}=-I_{2 i j}^{\sigma}$, allows us to write Eq. (16) in the following form:

$$
\begin{aligned}
\frac{I_{\mathrm{Fe}}^{\sigma}}{2 e / h}= & \sum_{i, j} \int_{-\infty}^{\infty} d \varepsilon \Gamma_{\sigma j i}^{S, S-1 / 2(s)}\left[f_{1<}(\varepsilon)-f_{2<}(\varepsilon-e V)\right] \\
& \times \operatorname{Im} G_{i j, i j}^{S, S-1 / 2(s)}(\varepsilon),
\end{aligned}
$$

where $\Gamma_{\sigma j i}^{S, S-1 / 2(s)}=2 \Gamma_{1 \sigma j i}^{S, S-1 / 2(s)} \Gamma_{2 \sigma j i}^{S, S-1 / 2(s)} /\left(\Gamma_{1 \sigma j i}^{S, S-1 / 2(s)}+\right.$ $\left.\Gamma_{2 \sigma j i}^{S, S-1 / 2(s)}\right)$ defines an effective broadening.

Then the conductance, in units of $G_{0}$ (the conductance quantum), measured with the tip over the Fe atom, and in the limit of low temperatures and small bias voltages, is given by

$$
G^{\mathrm{Fe}} / G_{0}=\sum_{\substack{\sigma, i=1-(2 S+1)^{*}(2 s+1), j=1-(2 S)^{*}(2 s+1)}} \Gamma_{\sigma j i}^{S, S-1 / 2,(s)} \operatorname{Im} G_{i j, i j}^{S, S-1 / 2,(s)}(\mathrm{eV}) .
$$

Finally, we can approximate $\Gamma_{\sigma j i}^{S, S-1 / 2,(s)}=$ $2 \eta_{\sigma j i}^{S, S-1 / 2,(s)} \Gamma_{1(\mathrm{Fe})}$ by assuming $\Gamma_{1} \ll \Gamma_{2}$. In the same way, the final expression for the conductance measured with the tip over the Co atom is

$$
G^{\mathrm{Co}} / G_{0}=\sum_{\substack{\sigma, i=1-(2 S+1)^{*}(2 s+1), j=1-(2 S+1)^{*}(2 s)}} \Gamma_{\sigma j i}^{s, s-1 / 2,(S)} \operatorname{Im} G_{i j, i j}^{s, s-1 / 2,(S)}(\mathrm{eV}) .
$$

\section{RESULTS AND DISCUSSION}

Our proposal, expressed through Hamiltonian (9), is that inelastic excitations observed in the electronic transport through magnetic atoms can be explained by a cotunneling process in which an electron is transferred from the tip (lead) to the atom and, in a second step, another electron is transferred from the atom to the lead (tip) [18,21]. In this process the total spin of the atom has fluctuated, first from $S$ to $S-1 / 2$ (Fe case), and then from $S-1 / 2$ to $S$ again, so that the final state is different from the initial one if the $M$ component is changed (see the Appendix). For very low temperatures we have an initial ground state and a final excited state; in such a case, a conductance step appears at a bias voltage equal to the excitation energy. A Kondo resonance will also appear for a manifold degenerate ground state, when the fluctuations among them involve a change in the spin projection equal to 1 , which corresponds to a spin flip of a conduction electron. All these features are captured in our model by the self-energy terms appearing in Eq. (13).

In our calculations (see Ref. [21]), we use a flatband approximation for the metal with a half bandwidth of $\Lambda=10 \mathrm{eV}$, and the values $\Delta^{\prime}=\varepsilon_{S=2}-\varepsilon_{S=3 / 2}=$ $2 \mathrm{eV}, \Gamma_{2(\mathrm{Fe})}=32 \mathrm{meV}, \Delta=\varepsilon_{S=3 / 2}-\varepsilon_{S=1}=1 \mathrm{eV}, \Gamma_{2(\mathrm{Co})}=$ $40 \mathrm{meV}$. The parameters of Hamiltonian (3) are extracted from Ref. [13], $D_{\mathrm{Fe}}=-1.53 \mathrm{meV}, E_{\mathrm{Fe}}=0.31 \mathrm{meV}, D_{\mathrm{Co}}=$ $2.7 \mathrm{meV}, E_{\mathrm{Co}}=0.0 \mathrm{meV}, g_{\mathrm{Fe}}=2.11$, and $g_{\mathrm{Co}}=2.16$. The value of the exchange interaction, $J=0.13 \mathrm{meV}$, is also taken from the work of Otte et al. [13], although another value, 0.19 $\mathrm{meV}$, will be explored below.

It is convenient to start considering the simplest case: $J=0$ and $D_{\mathrm{Fe}}=E_{\mathrm{Fe}}=D_{\mathrm{Co}}=0$, corresponding to having neither anisotropy terms $\left(H_{\text {atom }}^{\mathrm{Fe}}+H_{\text {atom }}^{\mathrm{Co}}=H_{\mathrm{atom}}^{\mathrm{Fe}(0)}+H_{\text {atom }}^{\mathrm{Co}(0)}\right)$ 


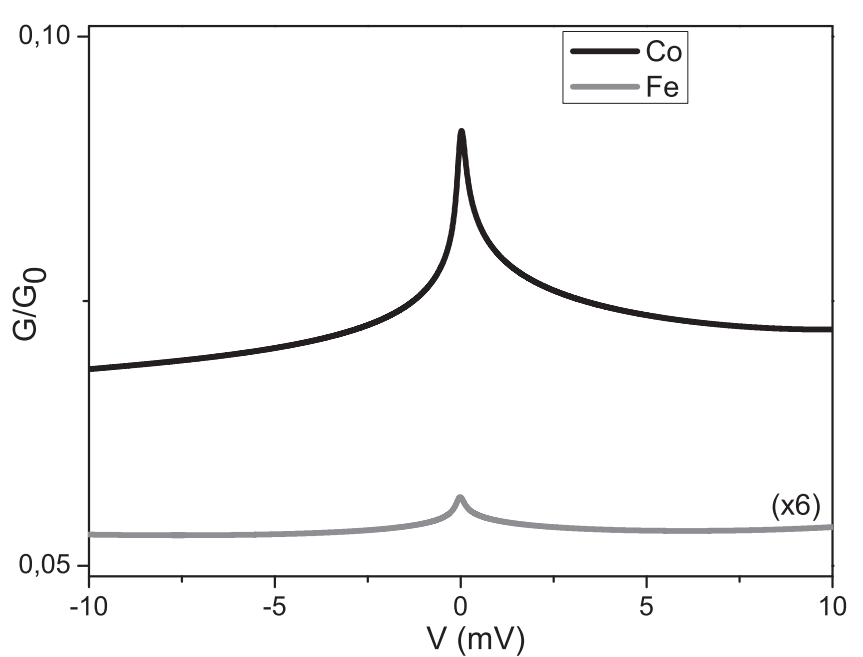

FIG. 2. Differential conductance as a function of the applied bias voltage for isolated atoms: Co (black curve) and Fe (gray curve, augmented by a factor of 6). In this case, the noninteracting atoms are not perturbed by crystalline and external magnetic fields.

nor exchange interaction between atoms. In this situation, $\psi_{i}^{S=2, s=3 / 2}=|S, M ; s, m\rangle$, there is a fivefold degenerate ground state for $\mathrm{Fe}$ and a fourfold degenerate ground state for Co. Kondo resonances appear in $\mathrm{Fe}$ or $\mathrm{Co}$, as can be seen in Fig. 2, because in both atoms, we find fluctuations among the many-fold degenerate ground state that involve changes in the spin projection equal to 1 . On the other hand, we cannot expect in this case to have conductance steps, because the cotunneling processes do not imply energy changes in the atom states. As we shall discuss below, introducing the anisotropy terms and the exchange interaction changes these Kondo resonances, in such a way that these peaks evolve into other resonances and /or conduction steps associated with changes in the atomic state energies. We see below that the practically constant values of the conductance around $V= \pm 10 \mathrm{mV}$ in both atoms, $\mathrm{Co}$ and $\mathrm{Fe}$, are the same when the anisotropy terms and the exchange interaction between atoms are introduced.

Going a step further, we introduce the anisotropy terms but taking $J=0$. There are now anisotropy terms in both atoms but the exchange interaction between them is zero. From the diagonalization of $H_{\text {atom }}^{\mathrm{Fe}}+H_{\text {atom }}^{\mathrm{Co}}$, the following doublet ground state, expanded in the basis set of states $|S, M ; s, m\rangle$, is obtained:

$$
\begin{aligned}
\psi_{1}^{S=2, s=3 / 2}= & 0.60|2,-2 ; 3 / 2,-3 / 2\rangle-0.35|2,-2 ; 3 / 2,1 / 2\rangle \\
& -0.14|2,0 ; 3 / 2,-3 / 2\rangle+0.08|2,0 ; 3 / 2,1 / 2\rangle \\
& +0.60|2,2 ; 3 / 2,-3 / 2\rangle-0.35|2,2 ; 3 / 2,1 / 2\rangle
\end{aligned}
$$

$$
\begin{aligned}
\psi_{2}^{S=2, s=3 / 2}= & 0.35|2,-2 ; 3 / 2,-1 / 2\rangle-0.60|2,-2 ; 3 / 2,3 / 2\rangle \\
& -0.08|2,0 ; 3 / 2,-1 / 2\rangle+0.14|2,0 ; 3 / 2,3 / 2\rangle \\
& +0.35|2,2 ; 3 / 2,-1 / 2\rangle-0.60|2,2 ; 3 / 2,3 / 2\rangle
\end{aligned}
$$
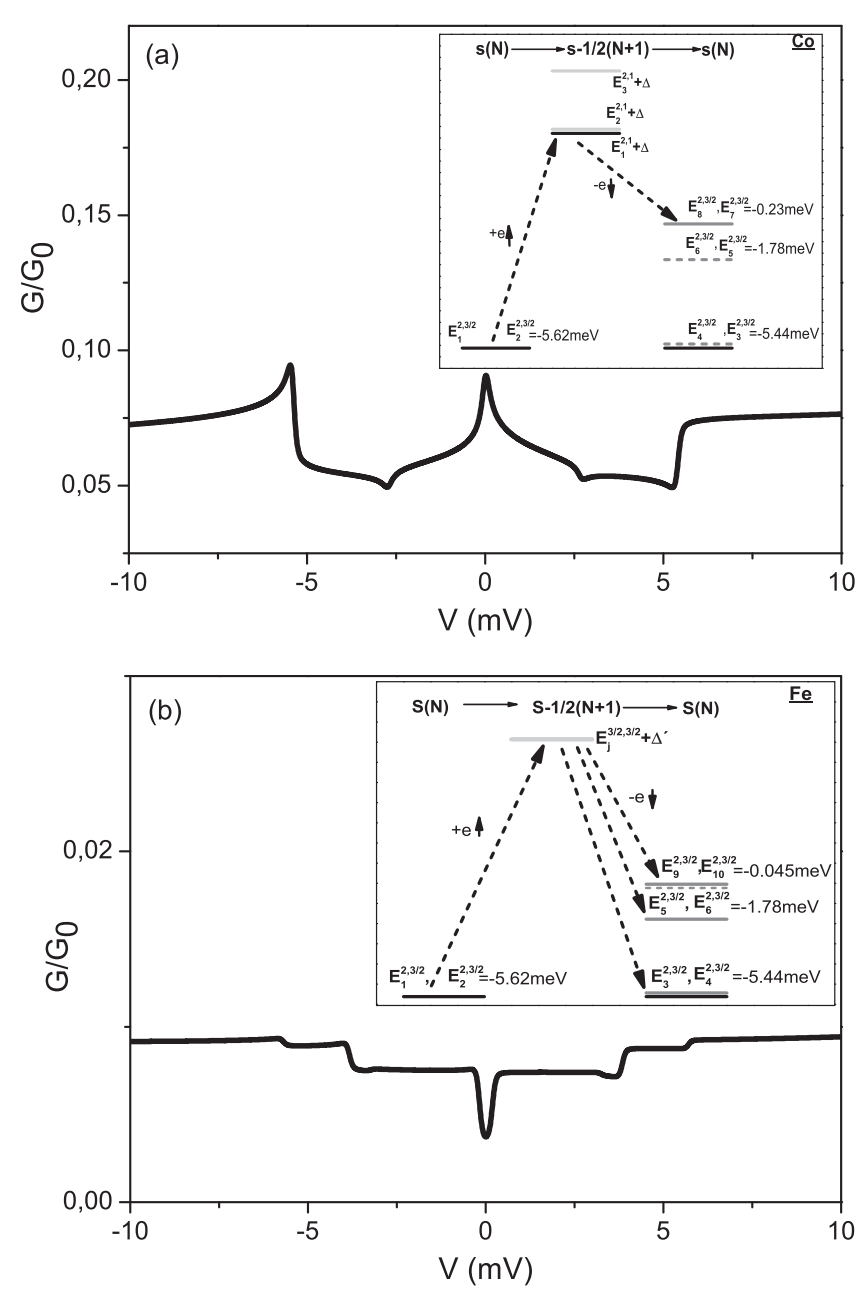

FIG. 3. Differential conductance as a function of the applied bias voltage for isolated atoms, including the crystalline field terms [see Eq. (3) in the text]: (a) Co and (b) Fe. The total energy eigenvalues corresponding to $\mathrm{N}$ and $(\mathrm{N}+1)$ electrons in the atoms are indicated in the insets.

as well as the following doublet first excited states, having an excitation energy of $0.18 \mathrm{meV}$ :

$$
\begin{aligned}
\psi_{3}^{S=2, s=3 / 2}= & -0.61|2,-2 ; 3 / 2,-3 / 2\rangle+0.35|2,-2 ; 3 / 2,1 / 2\rangle \\
& +0.61|2,2 ; 3 / 2,-3 / 2\rangle-0.35|2,2 ; 3 / 2,1 / 2\rangle,
\end{aligned}
$$

$$
\begin{aligned}
\psi_{4}^{S=2, s=3 / 2}= & 0.35|2,-2 ; 3 / 2,-1 / 2\rangle-0.61|2,-2 ; 3 / 2,3 / 2\rangle \\
& -0.35|2,2 ; 3 / 2,-1 / 2\rangle+0.61|2,2 ; 3 / 2,3 / 2\rangle .
\end{aligned}
$$

Eigenfunctions (20a) and (21a) are associated with a Kondo resonance in the $\mathrm{Co}$ atom, since changes $\Delta m= \pm 1$ in the spin projection occur. This is so because for $J=0$, the dimer behaves as two independent atoms, so that the differential conductance for the tip on top of $\mathrm{Fe}(\mathrm{Co})$ is the same as the one obtained for the isolated $\mathrm{Fe}(\mathrm{Co})$ atom. Consequently, we find the differential conductance shown in Fig. 3, in agreement with our previous results of Ref. [21]. In particular, for Co there is only one possible inelastic conductance channel corresponding to an excitation energy of $5.39 \mathrm{meV}$ [see Fig. 3(a) and its inset]. 
In the case of $\mathrm{Fe}$, the three inelastic channels indicated in the inset of Fig. 3(b) appear in the conductance spectrum at the following energies: 0.18, 3.84, and $5.57 \mathrm{meV}$ [see Fig. 3(b)]. The inelastic channel closest to the Fermi level, of $0.18 \mathrm{meV}$, is associated with the excitations to the eigenstates (20b) and (21b).

Consider now the case with the $J S \cdot s$ interaction included $(J=0.13 \mathrm{meV})$. Then, due to the $J$ coupling, the doublet ground states result in practically a combination of the states (20a) and (20b) or of the states (21a) and (21b):

$$
\begin{aligned}
\psi_{1}^{S=2, s=3 / 2}= & 0.14|2,-2 ; 3 / 2,-3 / 2\rangle-0.09|2,-2 ; 3 / 2,1 / 2\rangle \\
& +0.01|2,-1 ; 3 / 2,-1 / 2\rangle-0.01|2,-1 ; 3 / 2,3 / 2\rangle \\
& -0.12|2,0 ; 3 / 2,-3 / 2\rangle+0.07|2,0 ; 3 / 2,1 / 2\rangle \\
& -0.03|2,1 ; 3 / 2,-1 / 2\rangle+0.03|2,1 ; 3 / 2,3 / 2\rangle \\
& +0.86|2,2 ; 3 / 2,-3 / 2\rangle-0.45|2,2 ; 3 / 2,1 / 2\rangle,
\end{aligned}
$$

$$
\begin{aligned}
\psi_{2}^{S=2, s=3 / 2}= & -0.45|2,-2 ; 3 / 2,-1 / 2\rangle+0.86|2,-2 ; 3 / 2,3 / 2\rangle \\
& +0.03|2,-1 ; 3 / 2,-3 / 2\rangle-0.03|2,-1 ; 3 / 2,1 / 2\rangle \\
& +0.07|2,0 ; 3 / 2,-1 / 2\rangle-0.12|2,0 ; 3 / 2,3 / 2\rangle \\
& -0.01|2,1 ; 3 / 2,-3 / 2\rangle+0.01|2,1 ; 3 / 2,1 / 2\rangle \\
& -0.09|2,2 ; 3 / 2,-1 / 2\rangle-0.14|2,2 ; 3 / 2,3 / 2\rangle .
\end{aligned}
$$

Similarly, the doublet first excited states are also practically a combination of the states (20a) and (20b) or (21a) and (21b):

$$
\begin{aligned}
\psi_{3}^{S=2, s=3 / 2}= & 0.08|2,-2 ; 3 / 2,-1 / 2\rangle-0.16|2,-2 ; 3 / 2,3 / 2\rangle \\
& +0.05|2,0 ; 3 / 2,-1 / 2\rangle-0.09|2,0 ; 3 / 2,3 / 2\rangle \\
& -0.01|2,1 ; 3 / 2,-3 / 2\rangle+0.02|2,1 ; 3 / 2,1 / 2\rangle \\
& -0.53|2,2 ; 3 / 2,-1 / 2\rangle+0.82|2,2 ; 3 / 2,3 / 2\rangle,
\end{aligned}
$$

$$
\begin{aligned}
\psi_{4}^{S=2, s=3 / 2}= & 0.82|2,-2 ; 3 / 2,-3 / 2\rangle-0.53|2,-2 ; 3 / 2,1 / 2\rangle \\
& +0.02|2,-1 ; 3 / 2,-1 / 2\rangle-0.01|2,-1 ; 3 / 2,3 / 2\rangle \\
& -0.09|2,0 ; 3 / 2,-3 / 2\rangle+0.05|2,0 ; 3 / 2,1 / 2\rangle \\
& -0.16|2,2 ; 3 / 2,-3 / 2\rangle+0.08|2,2 ; 3 / 2,1 / 2\rangle .
\end{aligned}
$$

The ground state is a doublet where the $z$ component of the Co spin is mostly antiparallel to the Fe spin. The possibility of having $\Delta m= \pm 1$ fluctuations in the Co atom anticipates a Kondo peak for the tip on this atom. But we also have fluctuations $\Delta M= \pm 1$ in Fe, with smaller weights than in the case of $\mathrm{Co}$; then, it is possible to have a rather small but non-negligible Kondo resonance when the tip is over the $\mathrm{Fe}$ atom. These Kondo resonances can be seen in the conductance spectra shown in Fig. 4.

A very good agreement with the experimental trends [13] is obtained in this case $(B=0 \mathrm{~T})$. The most important changes in the differential conductance introduced by the antiferromag-
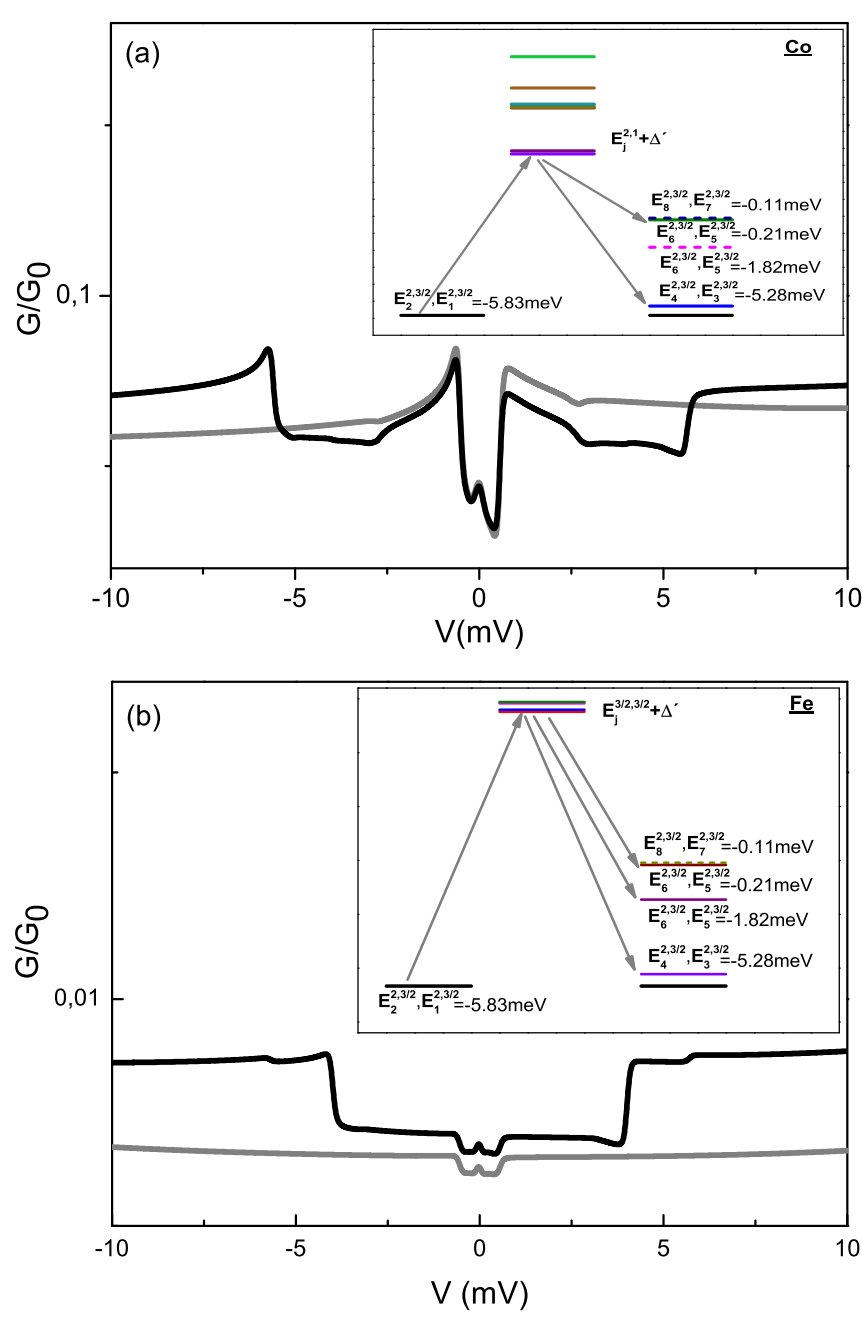

FIG. 4. (Color online) Differential conductance as a function of the applied bias voltage, for the tip on (a) the Co atom or (b) the $\mathrm{Fe}$ atom for a Fe-Co dimer $(J=0.13 \mathrm{meV})$. The gray curves correspond to the reduced configurational space (see the text).

netic interaction between $\mathrm{Fe}$ and $\mathrm{Co}$ appear around the Fermi level. In Co the Kondo peak is drastically reduced and a new two-step structure appears around the zero energy, reminiscent of the one found for the isolated $\mathrm{Fe}$, due to the mixing of the $\mathrm{Fe}$ and Co states. In the case of Fe, apart from the small Kondo peak, the intensity of the lowest-energy conductance steps is notably diminished. All these effects can be traced back to the changes in the wave functions (22) and (23). The very small Kondo resonance calculated with the tip on the Fe atom is not seen experimentally, which might be due to the energy resolution of the experiments.

The conductance steps appearing at larger energies are similar to the ones found for the isolated atoms and are associated with the different inelastic channels. For Co, one conductance step appears at $5.6 \mathrm{meV}(5.4 \mathrm{meV}$ in the isolated atom), while for $\mathrm{Fe}$, we find steps at 4.0 and $5.6 \mathrm{meV}$ (3.8 and $5.5 \mathrm{meV}$ in the isolated atom). See the insets of Fig. 4.

In view of these results, we have explored the possibility of using a reduced configurational space to analyze the dimer properties around the Fermi energy. In this approximation we have included only the ground [Eqs. (22a) and (23a)] and first 
excited doublet states [Eqs. (22b) and (23b)], and the four lowest-energy states with one electron more corresponding to either the spin configuration $S=3 / 2, s=3 / 2$ (tip on $\mathrm{Fe}$ ) or $S=2, s=1$ (tip on Co). Figure 4 also shows the differential conductance with this reduced basis set; the good description found around the Fermi level is encouraging for future treatments of larger-size clusters of similar atoms [26,27].

The conductance behavior for the interacting dimer in the presence of an applied magnetic field in the $z$ direction is shown in Fig. 5. We find as in the experiment [13], that the magnetic field initially reduces the splitting of the Kondo peak and at a field of approximately $2 \mathrm{~T}$, the two peaks merge into a Kondo peak similar to the one for an isolated Co atom. For a magnetic field $B_{z}=2 \mathrm{~T}$ the ground state again becomes a doublet with very appreciable weights of fluctuations $\Delta m= \pm 1$ in the Co
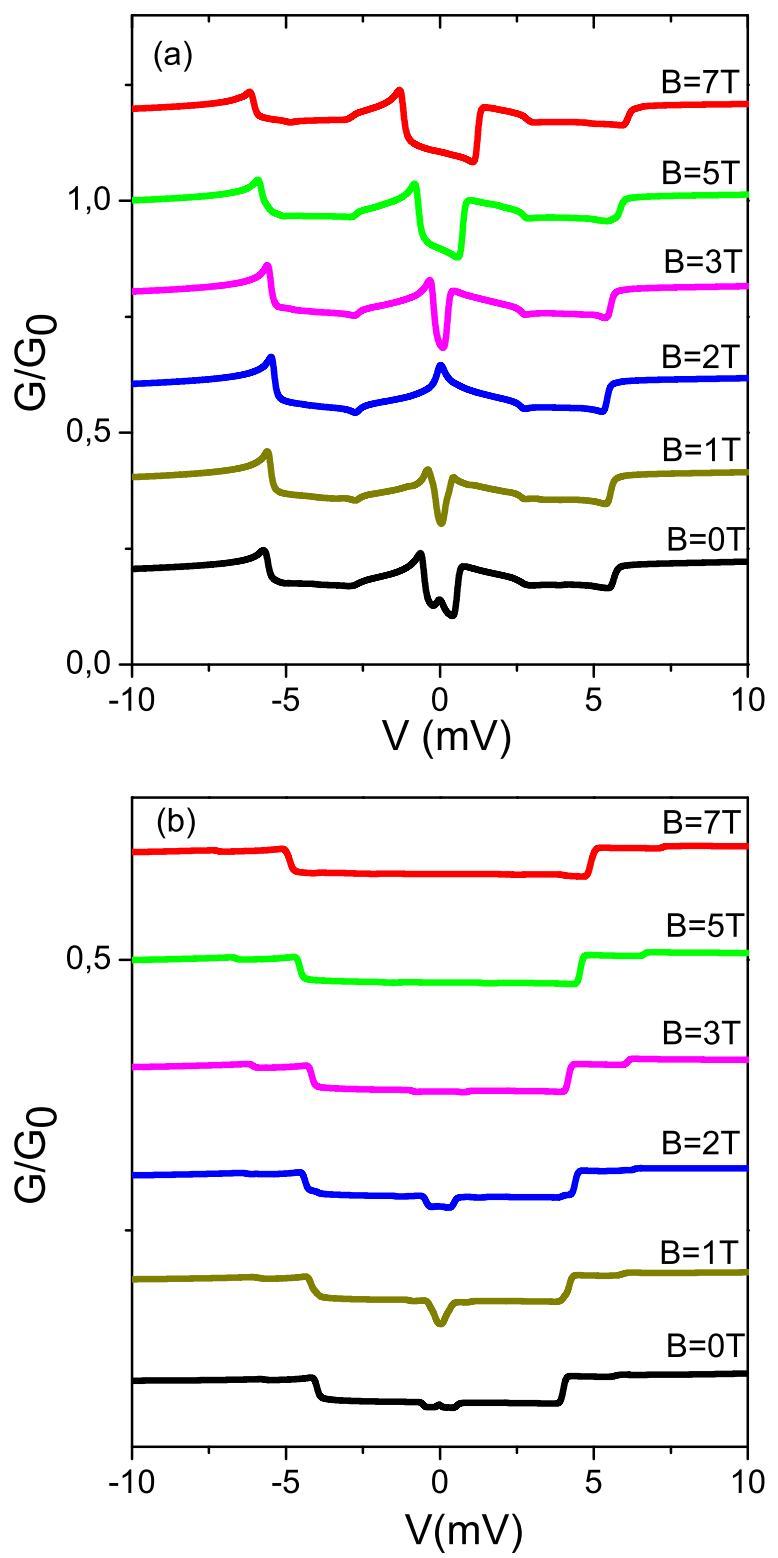

FIG. 5. (Color online) Conductance spectra dependence with the applied magnetic field in the $z$ direction (see Fig. 1) for Co (a) and $\mathrm{Fe}(\mathrm{b})$. The exchange interaction is $0.13 \mathrm{meV}$. atom. The two degenerated lowest-energy eigenstates with $S=2$ and $s=3 / 2$ are in this case

$$
\begin{aligned}
\psi_{1}^{S=2, s=3 / 2} \approx & -0.49|2,-2 ; 3 / 2,-1 / 2\rangle \\
& +0.86|2,-2 ; 3 / 2,3 / 2\rangle, \\
\psi_{2}^{S=2, s=3 / 2} \approx & -0.84|2,-2 ; 3 / 2,-3 / 2\rangle \\
& +0.49|2,-2 ; 3 / 2,1 / 2\rangle .
\end{aligned}
$$

The first one (24) corresponds to the Co spin being mostly antiparallel to the Fe spin, while the second one (25), to the Co spin mostly parallel to the Fe spin. It is found that the ground state changes from a predominantly antiferromagnetic (AF) character for magnetic fields below $2 \mathrm{~T}$ to a predominantly ferromagnetic (F) character for magnetic fields larger than this value. Interestingly, at the Kondo resonance, the two behaviors, $(\mathrm{AF})$ and $(\mathrm{F})$, coexist.

In Fig. 6 we show the behavior of the conductance through Co close to the Fermi level when the magnetic field $B_{z}$ is varied around $2 \mathrm{~T}$. The case of an isolated Co atom $(J=0)$ for a field of $0.4 \mathrm{~T}$ applied in the easy magnetization plane is also shown in Fig. 6. It is observed that the conductance spectrum in this case practically coincides with the one for the interacting dimer $(J=0.13 \mathrm{meV})$ in the presence of a magnetic field equal to $2.5 \mathrm{~T}$. The resemblance between both curves supports the observation of Otte et al. [13], that the exchange coupling to the Fe atom can be viewed as an effective field of $2.1 \mathrm{~T}$ opposing the external magnetic field. The observed agreement between the widths of the two peaks around the Fermi energy in both cases indicates that the strength of the Kondo screening interaction is not significantly affected by the exchange coupling.

On the other hand, by varying the value of the exchange interaction, we find that for $J=0.19 \mathrm{meV}$, the Kondo peak in Co is recovered at $B_{z}=3 \mathrm{~T}$, as it can be seen in Fig. 7(a). We also find that the conductance spectrum for an applied field $B_{z}=3.5 \mathrm{~T}$ in the case of $J=0.19 \mathrm{meV}$ practically reproduces the one for an isolated Co atom, and at $B_{z}=0.4 \mathrm{~T}$,

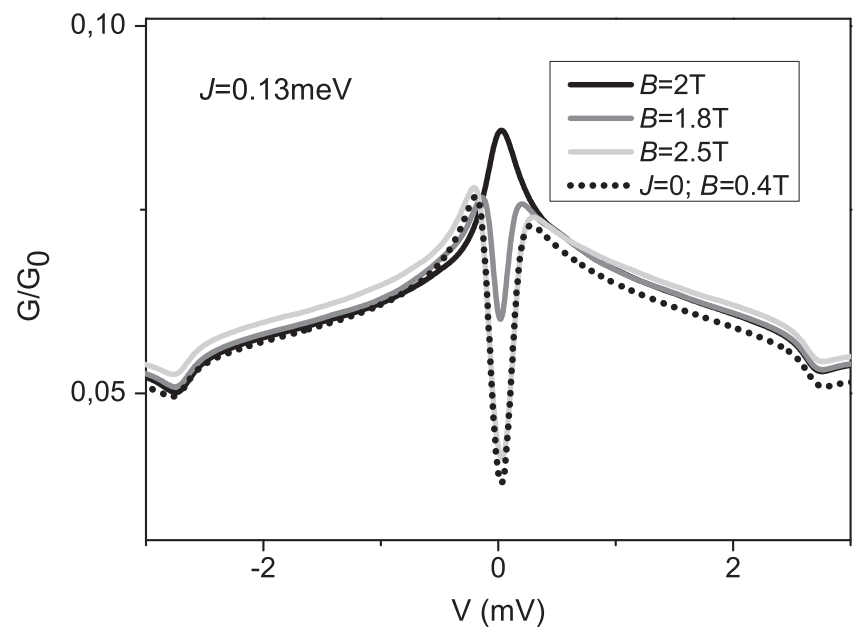

FIG. 6. Behavior of the conductance through the Co atom with the magnetic field applied in the $z$ direction around the value $B_{z}=2 \mathrm{~T}$ and for $J=0.13 \mathrm{meV}$. The dotted line corresponds to an isolated Co atom and $B_{z}=0.4 \mathrm{~T}$. 

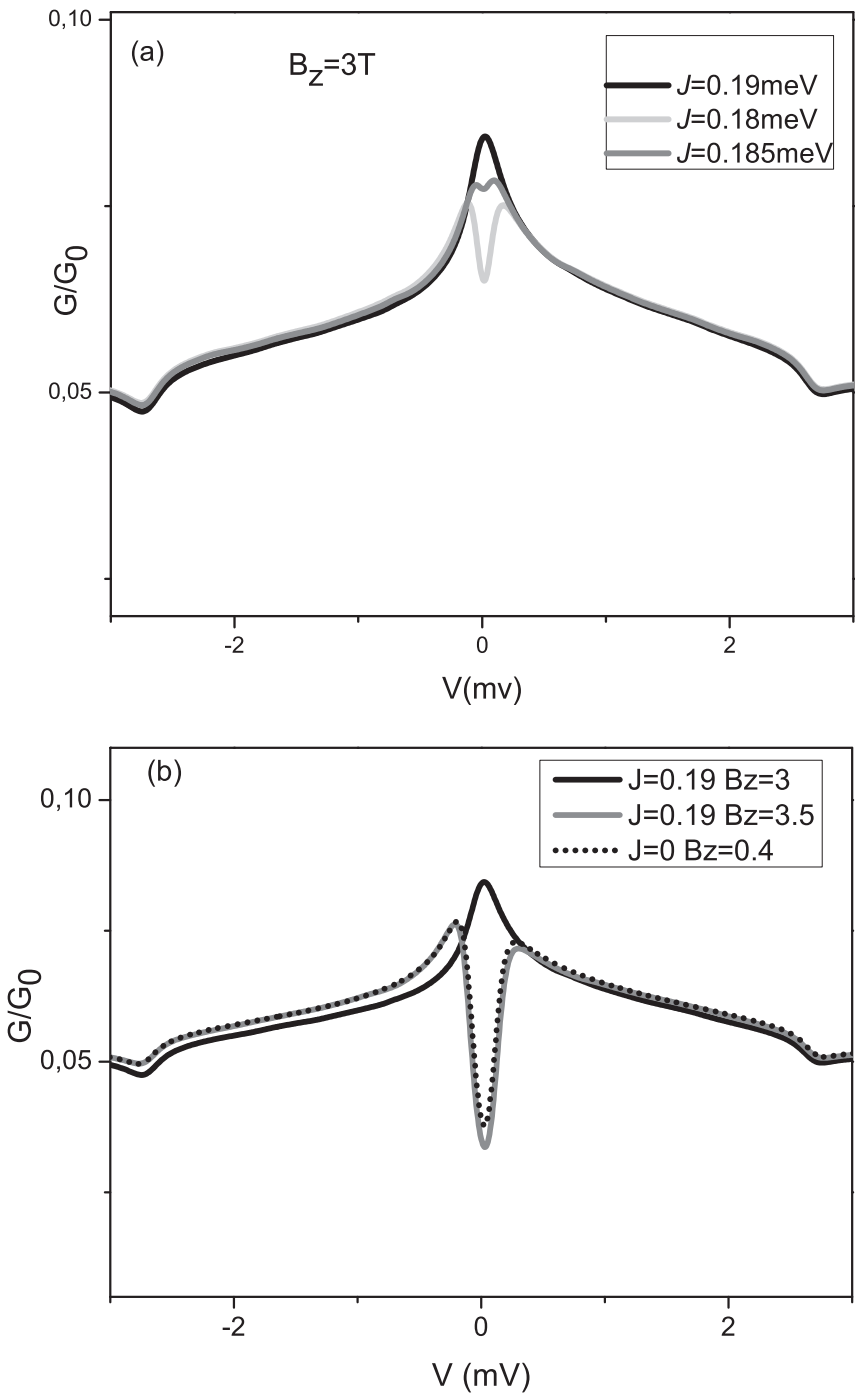

FIG. 7. (a) Evolution of the conductance through a Co atom with exchange interaction $J$ in the presence of a magnetic field $B_{z}=$ $3 \mathrm{~T}$. (b) Behavior of the conductance through a Co atom with the magnetic field applied in the $z$ direction and for $J=0.19 \mathrm{meV}: B_{z}=$ $3 \mathrm{~T}$ (black line), $B_{z}=3.5 \mathrm{~T}$ (gray line). The dotted line corresponds to an isolated Co atom and $B_{z}=0.4 \mathrm{~T}$.

reinforcing the idea of an effective field of $2.1 \mathrm{~T}$ opposing the external magnetic field, originating by the interaction with the Fe atom [Fig. 7(b)]. One might expect that taking this value of the exchange interaction, $J=0.19 \mathrm{meV}$, would yield a better agreement with the experiments; accordingly, we show in Fig. 8 the conductance spectra for $J=0.19 \mathrm{meV}$ and different values of $B_{z}$. A comparison between Figs. 5 and 8 indicates, however, that an overall agreement with the experiments [13] is better achieved by taking $J=0.13 \mathrm{meV}$.

Finally, we show in Fig. 9 the differential conductance for a magnetic field applied in the $x$ direction (see Fig. 1), taking $J=0.13 \mathrm{meV}$. We find in the case of Co that the Kondo peak is not recovered when the magnetic field increases, in agreement with the experimental results [13]. The lowestenergy conductance step in the case of $\mathrm{Fe}$ is found to follow the same evolution as in the experiments. The intensity of this step increases up to a magnetic field of around $3 \mathrm{~T}$, and then
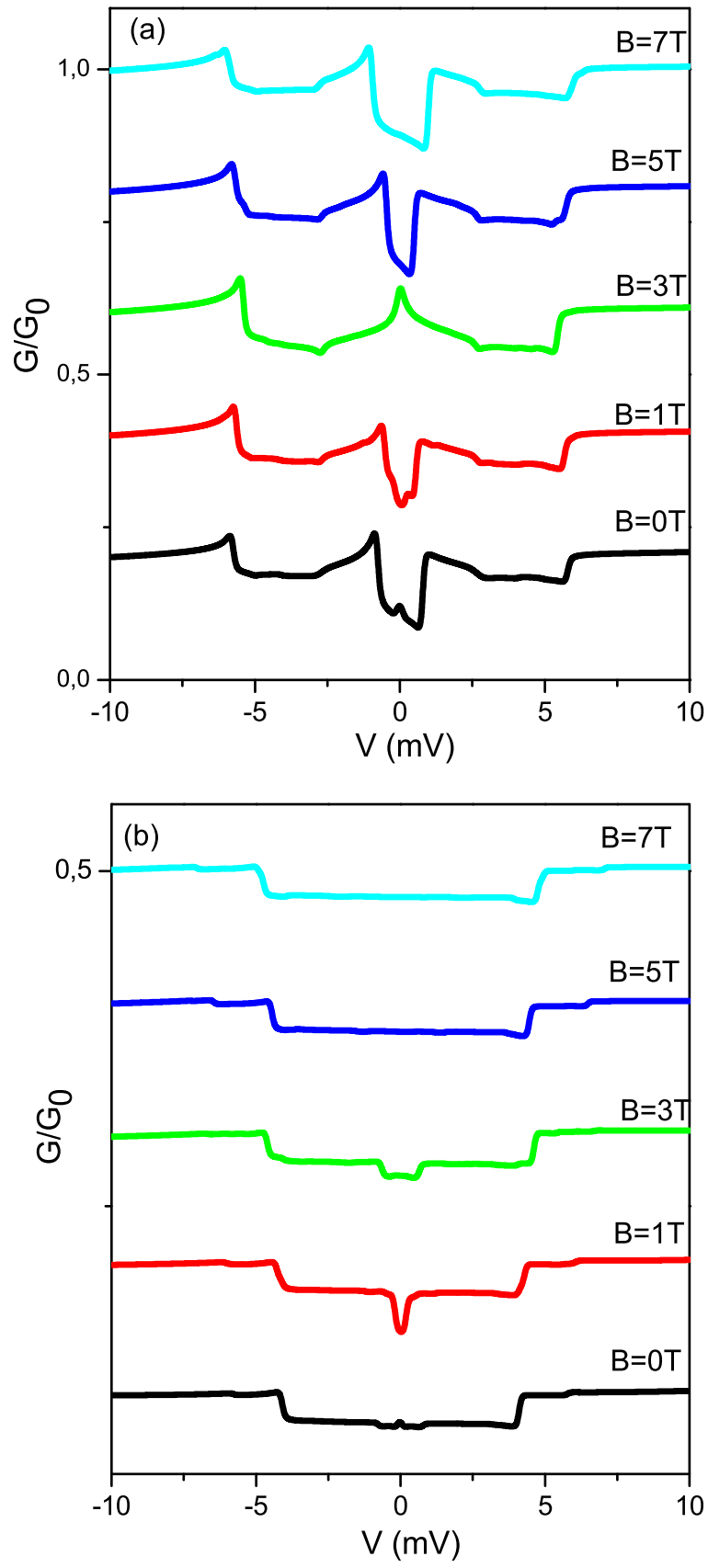

FIG. 8. (Color online) The same as in Fig. 5 for $J=0.19 \mathrm{meV}$.

it decreases as $B_{x}$ increases. For Co, our theoretical results show a very small structure close to the Fermi level, either a Kondo peak at zero field or a small depression for an applied magnetic field; although these very small features are not seen experimentally, these results are consistent with our calculated first excitation energy $(0.05,0.11,0.12$, and $0.08 \mathrm{meV}$ for $B_{x}=1,3,5$, and $7 \mathrm{~T}$, respectively). Figure 10 shows a close-up of the differential conductance for Co around the Fermi energy; notice here the width of the narrow features just mentioned.

\section{CONCLUSIONS}

In conclusion, we have analyzed the inelastic electron excitations in a $\mathrm{Fe} / \mathrm{Co}$ dimer deposited on $\mathrm{Cu}_{2} \mathrm{~N}$, as 

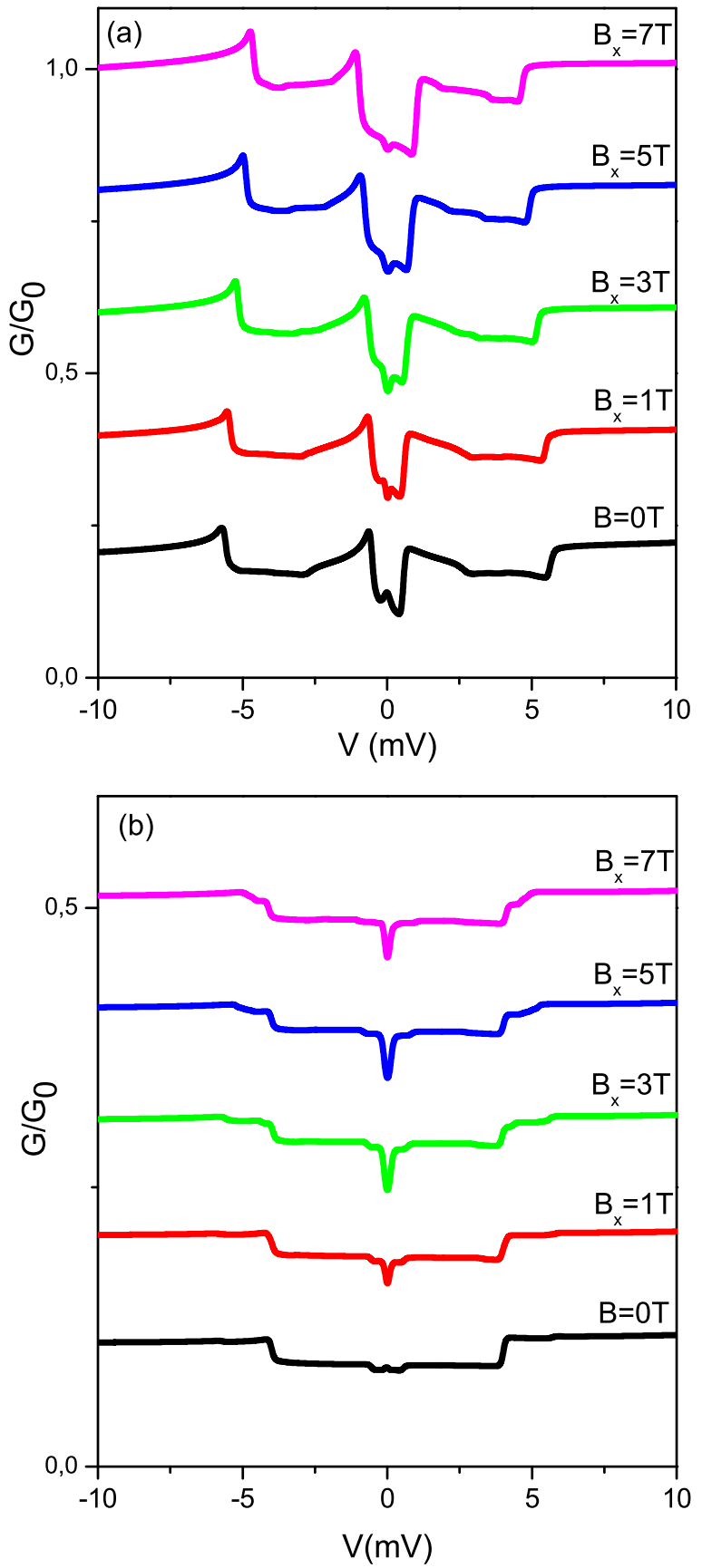

FIG. 9. (Color online) The same as in Fig. 5 for a magnetic field applied in the $x$ direction (see Fig. 1).

characterized by the differential conductance measured with a STM tip located on either Co or Fe [13]. In order to present a comprehensive understanding of the physics of this problem, we have also discussed initially the case of the independent atoms of $\mathrm{Fe}$ or $\mathrm{Co}$. In all these cases, the starting point is an ionic Hamiltonian that takes into account the atomic spin-flip fluctuations by means of a cotunneling process in which electrons are transferred to/from the metal. For the dimer case we introduce an appropriate basis set to take into account the $\mathrm{Fe} / \mathrm{Co}$ coupling. Then, in this basis set an equation-of-motion method allows us to calculate the Green's functions of the problem and the differential conductance

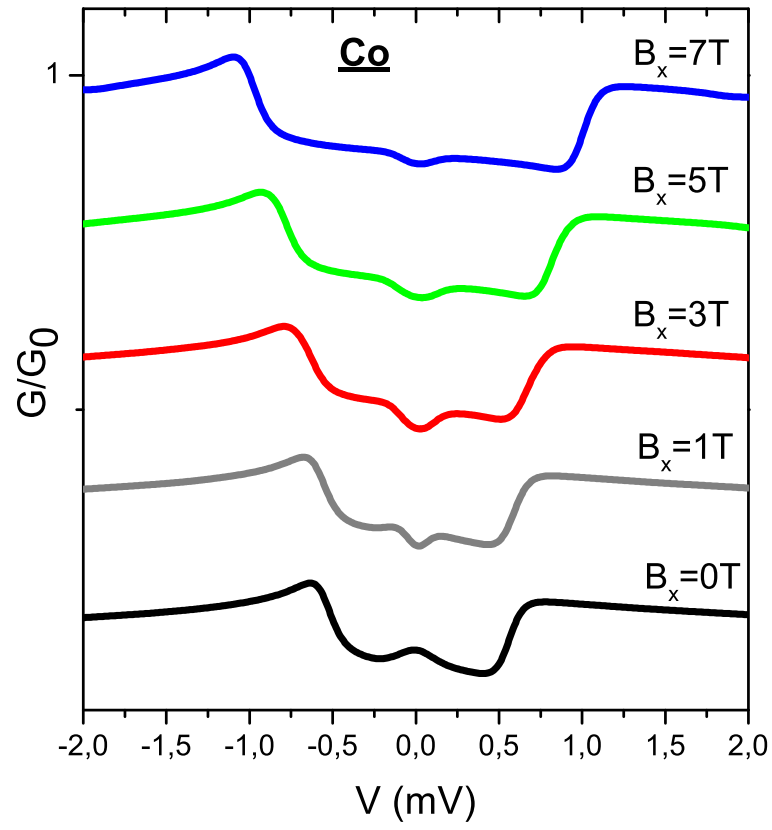

FIG. 10. (Color online) As Fig. 9 (Co), in the range $[-2.0,2.0]$ meV.

of the tip/Fe-Co/metal system. Our results show that the antiferromagnetic coupling of the two atoms substantially reduces the Kondo resonance that is found for the isolated Co atom. We also show that with a magnetic field perpendicular to the dimer direction, the antiferromagnetic coupling is changed gradually into ferromagnetic coupling, and a Kondo resonance is recovered for a magnetic field between 2 and $3 \mathrm{~T}$. We show that in this new Kondo resonance the system has a mixed antiferro- ferromagnetic character. Finally, we mention that our results for the differential conductance of the dimer system are in good agreement with the experimental data of Otte et al. [13]. showing, in particular, around the Fermi energy most of the characteristics found in the experiments.

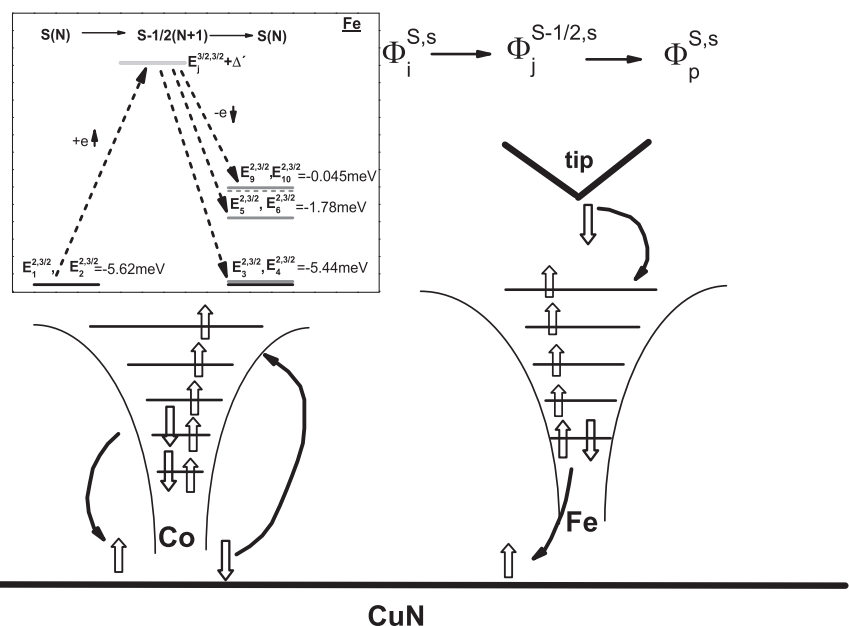

FIG. 11. The cotunneling process when the tip is over the Fe atom. In the inset we show schematically the excitation process mediated by the $S-1 / 2$ spin states of $\mathrm{Fe}$. 


\section{ACKNOWLEDGMENTS}

E.C.G. acknowledges financial support by ANPCyT through Grant No. PICT-2010-0294, CONICET through Grant No. PIP-201101-00621, and U.N.L. through CAI+D grants. F.F. has been supported by the Spanish MICIIN under Contract No. FIS2010-16046.

\section{APPENDIX}

In Fig. 11 we show schematically the electronic conduction occurring when the tip is over the Fe atom of the dimer Fe-Co: a spin-down electron from the tip is transferred to the atom, changing the total atomic spin from $S=2$ to $S=3 / 2$; then a spin-up electron is transferred from the atom to the surface and the total atomic spin changes again from $S=3 / 2$ to $S=2$. Along this process the dimer state has changed from the ground state $\Phi_{i}^{S, s}$ with total energy $E_{i}^{S, s}$ to the virtual state $\Phi_{j}^{S-1 / 2, s}$ with total energy $E_{j}^{S-1 / 2, s}$ and finally, to the excited state $\Phi_{p}^{S, s}$ with total energy $E_{p}^{S, s}$ (see the inset). Meanwhile, the Co atom spin is fluctuating due to the interaction with the surface: $s=3 / 2 \leftrightarrows s=1$
[1] C. F. Hirjibehedin, C. Y. Lin, A. F. Otte, M. Ternes, C. P. Lutz, B. A. Jones, and A. J. Heinrich, Science 317, 1199 (2007).

[2] A. F. Otte, M. Ternes, K. von Bergmann, S. Loth, H. Brune, C. P. Lutz, C. F. Hirjibehedin, and A. J. Heinrich, Nat. Phys. 4, 847 (2008).

[3] J. T. Li, W. D. Schneider, R. Berndt, and B. Delley, Phys. Rev. Lett. 80, 2893 (1998).

[4] M. Ternes, A. J. Heinrich, and W. D. Schneider, J. Phys.: Condens. Matter 21, 053001 (2009).

[5] J. J. Parks et al., Science 328, 1370 (2010)

[6] W. Liang, M. P. Shores, M. Bockralh, R. Long, and H. Park, Nature (London) 417, 725 (2002).

[7] C. Romeike, M. R. Wegewijs, W. Hofstetter, and H. Schoeller, Phys. Rev. Lett. 96, 196601 (2006).

[8] D. Goldhaber-Gordon, H. Shtrikman, D. Mahalu, D. AbuschMagder, U. Meirav, and M. A. Kastner, Nature (London) 391, 156 (1998).

[9] S. M. Cronenwett, T. H. Oosterkamp, and L. P. Kouwenhoven, Science 281, 540 (1998).

[10] H. B. Heersche, Z. de Groot, J. A. Folk, L. P. Kouwenhoven, and H. S. J. van der Zant, Phys. Rev. Lett. 96, 017205 (2006).

[11] F. H. L. Koppens, C. Buizert, K. J. Tielrooij, I. T. Vink, K. C. Nowack, T. Meunier, L. P. Kouwenhoven, and L. M. K. Vandersypen, Nature (London) 442, 766 (2006).

[12] R. Bulla, T. A. Costi, and T. Pruschke, Rev. Mod. Phys. 80, 395 (2008).
[13] A. F. Otte, M. Ternes, S. Loth, C. P. Lutz, C. F. Hirjibehedin, and A. J. Heinrich, Phys. Rev. Lett. 103, 107203 (2009).

[14] I. J. Hamad, L. Costa Ribeiro, G. B. Martins, and E. V. Anda, Phys. Rev. B 87, 115102 (2013).

[15] M. Persson, Phys. Rev. Lett. 103, 050801 (2009).

[16] A. Hurley, N. Baadji, and S. Sanvito, Phys. Rev. B 84, 115435 (2011).

[17] J. Fernández-Rossier, Phys. Rev. Lett. 102, 256802 (2009).

[18] F. Delgado and J. Fernandez-Rossier, Phys. Rev. B 84, 045439 (2011).

[19] J. Fransson, O. Eriksson, and A. V. Balatsky, Phys. Rev. B 81, 115454 (2010)

[20] N. Lorente and J. P. Gauyacq, Phys. Rev. Lett. 103, 176601 (2009).

[21] E. C. Goldberg and F. Flores, J. Phys.: Condens. Matter 25, 225001 (2013).

[22] Ph. Nozières and A. Blandin, J. Phys. 41, 193 (1980).

[23] E. C. Goldberg and F. Flores, Phys. Rev. B 77, 125121 (2008).

[24] L. V. Keldysh, Zh. Eksp. Teor. Fiz. 47, 1515 (1964) [Sov. Phys.JETP 20, 1018 (1965)].

[25] J. Hubbard, Proc. Roy. Soc. 285, 542 (1965).

[26] S. Loth, S. Baumann, C. P. Lutz, D. M. Eigler, and A. J. Heinrich, Science 335, 196 (2012).

[27] A. Spinelli, B. Bryant, F. Delgado, J. Fernández-Rossier, and A. F. Otte, Nature Mater. 13, 782 (2014). 